\title{
Prácticas evaluativas en el proceso de aprendizaje: estudio de caso
}

\author{
Núñez Guale Linda ${ }^{1}$ \\ Tigrero Suárez Freddy ${ }^{2}$ \\ Domo Mendoza Isauro ${ }^{3}$ \\ Valencia Medranda Adrian ${ }^{4}$
}

\section{Resumen}

El presente artículo tiene como propósito analizar las prácticas evaluativas en el proceso de aprendizaje de la carrera de administración de empresas de la Universidad Estatal Peninsula de Santa Elena. La metodología es de tipo descriptiva-analítica, de campo, no experimental y transeccional. Se estudiaron dos poblaciones, la primera, estuvo conformada por 621 estudiantes de la carrera de administración de empresa correspondiente al primero período académico 2018-2019, el tipo de muestreo aplicado fue no probabilístico por conveniencia, resultando una muestra de 175 estudiantes ubicados en el tercero y cuarto semestre de la carrera. La segunda población estuvo conformada por 28 docentes de planta, para lo cual se utilizó la técnica del censo. Se aplicaron encuestas mediante un cuestionario con respuestas cerradas diseñadas en tres categorías de valoración: nunca, en ocasiones, siempre. Para el procesamiento de la información se utilizaron métodos estadísticos multivariados: análisis de ordenamiento y clasificación. En los resultados destaca la transparencia en las calificaciones las cuales son del conocimiento de los estudiantes;

Recibido: 20.03.19 Aceptado: 20.06.19

1 Ingeniera Comercial, mención Gestión Empresarial; con Maestrías en Gerencia Educativa y Administración de Empresas, mención Recursos Humanos. Cursando estudios doctorales en Ciencias de la Administración. Diplomados en el área educativa y administrativa. Ejercicio profesional en calidad de Presidenta de Compañía, Decana de Facultad, Directora de Carrera, Coordinadora de Áreas Administrativas.

2 Licenciado en Administración Educativa. Maestría en Gerencia y Educación Superior. Coordinador de Currículo y Capacitación de Vicerrectorado Académico en UPSE. Docente Titular de Universidad Estatal Península de Santa Elena. Facilitador en áreas de educación y administrativas. Supervisor Provincial del Área de Educación Media Santa Elena.

3 Ingeniero Comercial, Abogado de los Tribunales y Juzgados de la República del Ecuador. Magíster en Administración de Empresas con mención en Marketing y Recursos Humanos. Cursando estudios doctorales en Ciencias del Derecho en Argentina. Docente en la facultad de Ciencias Administrativas de la Universidad Estatal Península de Santa Elena. Asesor Jurídico en la Empresa Publica Santa Elena E.P., Titular de consultorio Jurídico Práctica Judicial.

4 Ingeniero Comercial con Maestría en Administración y Dirección de Empresa. Diplomado Superior en Aplicación y Diseño de Modelos Educativos. Cursando estudios doctorales en Ciencias de la Administración en la República de Argentina. Docente Universitario. Docente Investigador acreditado por el Senescyt. Facilitador, Asesory Capacitador de empresas públicas y privadas. 
los docentes asumen que el proceso de evaluación busca, en primer lugar, una potenciación en el aprendizaje, asi como, la comprobación y verificación de lo aprendido. Desde la perspectiva de los estudiantes la evaluación se centra, principalmente, en la comprobación, verificación y medición de lo aprendido, dejando en tercer lugar la potenciación del aprendizaje, en contraposición con lo plateado por los profesores. Destaca que los resultados de los estudiantes reflejan un valor máximo del $61,78 \%$, disminuyendo apenas entre 1 o 2 puntos porcentuales en la ubicación ordinal. En las respuestas de los estudiantes existe marcada tendencia para la alternativa en ocasiones, identificando que no siempre están satisfechos con los rasgos del proceso evaluativo. Se concluye que existen dierencias entre estudiantes y profesores sobre la percepción del sistema de evaluación.

Palabras clave: practicas evaluativas, aprendizaje universitario, carrera universitaria, potencicación del aprendizaje, administración de empresas.

\title{
Evaluative practices in the learning process: case study
}

\begin{abstract}
The purpose of this article is to analyze the evaluation practices in the learning process of the business administration career of the Santa Elena Peninsula State University. The methodology is descriptive-analytical, field, non-experimental and transectional. Two populations were studied, the first one was made up of 621 students of the business administration career corresponding to the first academic period 2018-2019, the type of sampling applied was not probabilistic for convenience, resulting in a sample of 175 students located in the third and fourth semester of the race. The second population was made up of 28 plant teachers, for which the census technique was used. Surveys were applied through a questionnaire with closed answers designed in three assessment categories: never, sometimes, always. For the information processing, multivariate statistical methods were used: ordering and classification analysis. The results highlight the transparency in the grades which are known to the students; Teachers assume that the evaluation process seeks, in the first place, an empowerment in learning, as well as the verification and verification of what has been learned. From the perspective of the students, the evaluation focuses mainly on the verification, verification and measurement of what has been learned, leaving thirdly the empowerment of learning, as opposed to what is silvery by the teachers. It emphasizes that the results of the students reflect a maximum value of $61.78 \%$, decreasing only between 1 or 2 percentage points in the ordinal location. In the students' responses there is a marked tendency for the alternative at times, identifying that they are not always satisfied with the features of the evaluation process. It is concluded that there are differences between students and teachers on the perception of the evaluation system.
\end{abstract}

Keywords: evaluation practices, university learning, university career, learning potentiation, business administration. 


\section{Introducción}

La educación superior juega un importante papel en el desarrollo de lospaísesy sus comunidades; una educación de excelencia en una nación otorga más posibilidades de progreso; mientras más se adecuen los procesos de enseñanza a la realidad, más calificados y acoplados estarán los ciudadanos a este mundo globalizado y competitivo. A lo largo de la historia, la educación desde los niveles básicos hasta la formación profesional ha vivido grandes cambios a nivel mundial; experiencias propias y de benchmarking aplicadas por instituciones educativas hangenerado expectativas de progreso en diversos países de América Latina, Europa y en otros contextos.

En el proceso de enseñanzaaprendizaje la evaluación se constituye en uno de los elementos fundamentales para consolidar la formación y el conocimiento; no obstante, son variadas las estrategias de evaluación las cuales dependerán del paradigma educativo, desde el condusctismo hasta la teoría sociocultural, cada uno define el propósito de la educación y su correspondiente evaluación.

No obstante, la estandarización educativa ha vuelto obsoleto el currículum y ha lastrado las escuelas latinoamericanas con una burocracia despótica y arbitraria, cuya inflexibilidad está minando la adaptación al futuro. Estas viejas ideas del cambio educativo surgidas en el siglo $X X$ están totalmente desfasadas y no sirven para este rápido, flexible y vulnerable nuevo mundo del siglo XXI (Hargreaves y Shirley, 2012, 13)

Sin lugar a dudas, las prácticas evaluativas, dentro de las universidades, son un pilar esencial que permite al futuro profesional encontrarse desde las aulas de clases con la realidad de su campo laboral. Muchas instituciones de educación superior dedican parte de su planificación, junto a su equipo de docentes, para organizar las mejores experiencias a través de la aplicación de herramientas modernas y conductoras de resultados favorables para sus estudiantes, dejando de lado la tradicional recepción de exámenes plasmados en instrumentos ortodoxos.

Tomando en consideración lo anterior, se hace un recorrido teórico por los procesos de enseñanza y las prácticas evaluativas en latinoamerica y especialmente en Ecuador, para luego mostrar los resultados de un trabajo de campo que analizó las prácticas evaluativas de la Universidad Estatal Península de Santa Elena en la carrera de Administracion de empresas comparando la opinión de los profesores y los estudiantes.

Para tal fin, se ejecutó una investigación de tipo descriptiva durante el primer período2018 de la carrera de Administración de Empresas de la Facultad de Ciencias Administración, tomando como sujetos de estudio a docentes y alumnos. Se diseñó un instrumento con tres categorías de valoración: Nunca, En ocasiones, Siempre. Para el procesamiento de la información se utilizaron métodos estadísticos multivariados: análisis de ordenamiento y clasificación,los cuales permitieron abordar las variables de estudio, comprendiendo la percepción de los involucrados sobre tipos de evaluación, objetivos procedimientos, efectos, impacto y otras aristas propias de la formación profesional. 


\section{Proceso de enseñanza- aprendizaje y prácticas evaluativas. Reflexiones teóricas}

Del siglo XVIII al XIX, el desarrollo social y científico influyó notablemente en las teorías de la educación, con valiosas concepciones pedagógicas hechas por filósofos y pedagogos como: J.J. Rousseau, J.R. Pestalozzi, A. Diestenveg, M.V. Lomonosov, I.I. Bietskoi, L.N. Tolstoi, K.D. Ushinski, entre otros. Para finales del siglo $\mathrm{XIX}$ y principios del siglo $\mathrm{XX}$, diversas tendencias pedagógicas, psicológicas y didácticas han aportado diferentes paradigmas para mejorar el acto educativo y en específico la evaluación del aprendizaje, resaltando en cada una de ellas, los elementos psicológicos que intervienen en la educación: conductuales, afectivos, cognoscitivos o sociales (Balam, 2009)

El proceso de enseñanza aprendizaje se concreta en la exploración de los conocimientos y en la evaluación de los aprendizajes, correspondientemente, "las innovaciones introducidas en la evaluación inciden directamente en los objetivos y la metodología de enseñanzaaprendizaje". (Watts y García-Carbonell, citados en Ibarra y Rodríguez, 2010). En este sentido, ambos procesos se nutren para construir el sistema educativo de un país el cual será reflejo de las políticas de Estado y de los saberes de sus especialistas y líderes. Al respecto Ibarra y Rodríguez (2010), señala que la evaluación del aprendizaje se convierte en una característica de las instituciones educativas orientadas al desarrollo del alumnado y su aprendizaje.

Consecuentemente,

paradigmas educativos orientan estrategias de evaluación utilizadas en la práctica docente. En este sentido, desde el conductismo, la evaluación compara la actuación de una persona consigo misma y no con otras, o con respecto a una norma, tal como lo hacen las pruebas estandarizadas; siendo imprescindible utilizar instrumentos estructurados para constatar el logro de objetivos conductuales. Otro paradigma importante, es el cognitivo que enfatiza la transcendencia de evaluar habilidades del pensamiento y reforzamiento; mientras que el paradigma humanista concibe la visión del hombre como un ser creativo, libre y consciente, desde este enfoque, el objetivo de la educación es promover la autorealización y estimular las potencialidades del ser humano, y considera la autoevaluación como válida para el alumnado. (Balam, 2009)

Otros paradigmas, como el psicoanalítico propugnan una evaluación que da mayor prioridad al proceso que al resultado educativo; de manera general se logran los objetivos; pero, se identifican los elementos no esperados, para luego establecer la autoevaluación personal y colectiva, resultando una calificación final. Asimismo, desde la Teoría Paigetiana se critican los exámenes tradicionales porque fomenta la memorización y no se estimula el desarrollo de la inteligencia y buenos métodos de trabajo. Finalmente, la Teoría Socio-Cultural plantea una evaluación dirigida a determinar el nivel de desarrollo potencial y real de los estudiantes, es decir, evaluar productos;pero, también, procesos. Desde este enfoque no existe alejamiento entre estudiante y profesor en las pruebas. (Balam, 2009)

La evaluación se ha convertido, desde hace un tiempo, en la piedra angular del sistema educativo. No se puede comprender la enseñanza sin tener 
en cuenta la evaluación. No sin motivo, porque la evaluación condiciona todo el proceso de enseñanza-aprendizaje. (...) Una evaluación pobre da lugar a un proceso de enseñanza pobre. Una evaluación inmoral, corrompe la entraña de la actividad docente (Moreno, 2016)

Invariablemente, de acuerdo al paradigma que rige el proceso de enseñanza-aprendizaje privarán unas prácticas evaluativas antes que otras. En este sentido,la evaluación constituye un factor que condiciona decisivamente la enseñanza del profesor(Nieto, citado en Ibarra y Rodríguez, 2010). Determinar la estrategia de evaluación se convierte en un reto para medir los conocimientos adquiridos por los estudiantes y para lograr el proceso de aprendizaje integral.

Comúnmente, la evaluación se utiliza para comprobar los conocimientos de los estudiantes, estando más orientada a resultados que a procesos, descuidando aspectos importates que se evidencia durante el aprendizaje aun cuando no puedan ser medidos - evaluados a través de un solo instrumento. Razón por la cual, se empieza a enfatizar la necesidad de articular sistemas de seguimiento, lo que podría entenderse como evolución hacia opciones más formativas/ formadoras de la evaluación, aunque desde dos vertientes diferenciadas pero ni disociadas ni incompatibles. (Ibarra, y Rodríguez, 2010:395)

Mas allá, la evaluación, es consustancial al proceso educativo, es ilustrativa en el reconocimiento de la realidad. Su discurso puede obstaculizar, favorecer 0 disuadir cualquier innovación, al resaltar "la forma que los estudiantes se apropian y asimilan los nuevos conocimientos..." (Ahumada, 2001: 230), los resultados del proceso de evaluación describen la eficacia del sistema educativo asi como el alcance y logro de los objetivos trazados, es decir, los resultados son una evidencia del proceso, su éxito o fracaso describen el camino recorrido. (LLECE, 2008)

De manera general, la evaluación puede ser diagnóstica, sumativa 0 formativa, aun cuando sobre está última debería estar el mayor esfuerzo. La evaluación del aprendizaje es asumida como un momento final que interesa al profesor para comprobar qué tanto aprendieron sus alumnos (cuantificar su saber) y no como un proceso que persiga, sobre todo, mejorar y potenciar el aprendizaje de éstos cualitativamente y de manera integral, lo que hace que la evaluación quede limitada a unas pocas funciones y que se restrinja así el efecto formativo que ha de tener. (Hernández, 2016: 5)

La Organización de las Naciones Unidas para la Educación, la Ciencia y la Cultura señala que la evaluación formativa, es decir, aquella ocupada en asentar los conocimientos de manera integral, específicamente, asegura que este tipo de evaluación mejora los resultados de los estudiantes cuando realmente forma parte de un proceso "justo, válido y fiable de recopilación, interpretación y utilización de información generada a partir de métodos utilizados a lo largo del proceso de aprendizaje del estudiante, refiriendo, además, que su efectividad depende del uso, por parte de los docentes, de diferentes canales y normas de evaluación de alta calidad. La claridad de los objetivos de aprendizaje marcará la diferencia en el diseño del sistema de evaluación, asi como, de sus resultados. (UNESCO, 2018)

El proceso de evaluación formativa es eficaz cuando es parte de un sistema que garantiza que los docentes utilicen diferentes canales, planes y normas 
de evaluación de alta calidad. Estas prácticas deberían estar apoyadas por una política de evaluación escolar basada en datos objetivos. En este sentido, desde las concepciones formativas actuales, la evaluación debe registrar un grupo de cualidades que sirven a un fin fundamental: formar o educar y se entiende que es formativa toda evaluación que ayude al alumno a aprender y a desarrollarse, que sirva para corregir, regular, impulsar, mejorar y producir aprendizajes. (Álvarez, 2006; UNESCO, 2018).

Por su parte, Boud y Falchikov (citados en Ibarray Rodríguez, 2010) proponen una evaluación alternativa que prime la implicación del estudiante, a través de tareas auténticas y con posibilidad de una retroalimentación eficaz que suponga una posibilidad de cambio o mejora. Hasta ahora, predomina el concepto de evaluación tradicional respecto a quién diseña y realiza la evaluación (profesor). El profesorado, individual o colectivamente, a través de tribunales o comisiones, es el agente evaluador (más bien calificador). Por lo general, el alumnado no participa, son poco comunes estrategias como la autoevaluación o la coevaluación, es receptor de las consecuencias de la evaluación: la calificación, la superación de la asignatura y la obtención del título. (Ibarra y Rodríguez, 2010: 394)

Correspondientemente, los profesores deben desarrollar la capacidad de analizar y discriminar los conocimientos que deben ser evaluados por encima de otros. Los criterios son tan importantes como la adminsitración del conocimiento, es decir, el proceso de enseñanza aprendizaje se concreta en la capacidad que tiene el docente para lograr la participación activa de los alumnos en su aprendizaje, e incluso, en el diseño de los sistemas de evaluación, convertir a los estudiantes en partícipes de sus evaluaciones aumenta el compromiso y por ende favorece los resultados. Para TurpoGebera (2013:230) "Es imprescindible investigar qué, cómo, dónde, cuándo, por qué ocurre, y qué responsabilidad compete a los docentes. Su abordaje supone reducir el foco de atención, al proceso evaluativo desarrollado por los docentes, al discriminar, valorar, criticar, decidir entre lo que piensan que tiene un valor en sí y lo que carece de él".

Contrariamente, Barberá (2003), plantea la necesidad de incorporar una evaluación compleja que dependa estrictamente de la voluntad del profesor. Esta posibilidad supone el plantear a los alumnos tareas de evaluación complejas basadas en la toma de decisiones de cualquier área o disciplina, en contraposición a tareas de tipo más telegráfico que enuncian lo mínimo suficiente para ser resueltas. Se expresa en un enfoque progresivo, actividades de evaluaciones globalizadas y a la vez paulatinas en su secuencia interna. Una propuesta de encadenamiento de los instrumentos propuestos a lo largo de un semestre, llamado Programa Evaluativo que va de la mano del Enfoque de la Evaluación que representan el conjunto de pensamientos, creencias, ideas, etc. del profesor sobre la evaluación. Lo que abordaría un sentido de independencia del proceso de evaluación, la naturaleza de las tareas o actividades en cuanto a su interpretación se refiere.

Si se busca relacionar de forma significativa a la evaluación con la mejora de la escuela, es imperativo mirar a la evaluación a través de nuevos ojos. Los actuales sistemas de evaluación (tradicionales) son nocivos para un gran número de alumnos y ese daño 
surge, entre otras causas, del fracaso para balancear el uso de las pruebas estandarizadas y las evaluaciones de aula al servicio de la mejora escolar. (Moreno, 2016)

\section{Prácticas evaluativas en las universidades de Latinoamerica}

Desde la década de los noventa, diversos organismos internacionales señalaron la necesidad de replantear el rol del docente de educación superior, de suerte que pudiera responder mejor a las demandas de una sociedad globalizada e interconectada. Esto significa un firme compromiso de las instituciones de educación superior de asumir un nuevo paradigma de formación, basado en el aprendizaje a lo largo de la vida, la orientación prioritaria hacia el autoaprendizaje y el diseño de innovadoras modalidades educativas en las cuales el alumno es el actor central del proceso formativo (Barrón, 2009).

Las universidades junto a sus procedimientos, protocolos y normativas educativas deben incorporar bases formativas modernas donde sus educandos se conviertan en críticos del proceso de aprendizaje y no solo en cumplidores de instrumentos sumativos. Resaltar tendencias de inclusión en trabajos, prácticas, ensayos, experimentación, observación del campo profesional, etc., despertar el interés de su experticia desde el aula de clases, prepararse para la vida y su profesión, entre otras acciones que impacten, no solo para momentos puntuales de evaluación, sino, por el contrario, para crear procesos de aprendizajes que trasciendan el cumplimiento de una nota o el pase de un semestre. El compromiso es generar una reflexión crítica y la consolidación de los conocimientos.

Sobre lo anterior, el enfoque predominante de evaluación ha generado, a lo largo de los años, sujetos pasivos, es decir, los alumnos no tienen ningún otro papel que no sea someterse a los actos de evaluación de los demás, ser medidos y clasificados,cumplir con las normas y procedimientos para satisfacer las necesidades de una burocracia evaluadora y de manera automática responder a instrumentos de evaluación, en su mayoría, diseñados para producir una calificación (Boud, 2007, 17)

Por otro lado, los esfuerzos encaminados desde siglos atrás, han pretendido mejorar los procesos de aprendizaje en las universidades, por lo que se acompaña con elementos fundamentales de la psicología como ciencia que reconoce el comportamiento humano y su personalidad para abrir el conocimiento y estímulos potenciales.

En cuanto a las interrelaciones alrededor de las prácticas evaluativas actuales, estas se han tornado dominantes y opresoras, en algunos casos el docente es el dueño absoluto del saber, lo que puede limitar al estudiante al momento de reflexionar sus procesos de aprendizaje y sentirse responsable del conocimiento, por el miedo al castigo y a la represión manifiesta en bajas notas y desaprobaciones en los productos académicos derivados de los proyectos académicos de asignatura; también se evidencian acciones de enjuiciamiento sobre las características de la enseñanza, demostrados a partir de expresiones enmarcadas en rendimientos que dan cuenta de aprendizajes teóricos, expresados en pruebas memorísticas que no llevan a la retroalimentación crítica, aspecto 
dinamizador del acto pedagógico y componente integrador de la formación (Duque, Henao y Palacios, 2017)

Haciendo un recorrido por diferentes praticas evaluativas se pueden destacar las reseñadas por Duque, Henao \& Palacios (2017), aplicadas en los programas de Enfermería, Bacteriología y Administración de la Salud en la Facultad de Ciencias de la Salud, en el contexto de la educación superior de la Universidad Católica de Manizales, Colombia (cuadro 1):

\section{Cuadro 1 \\ Prácticas Evaluativas}

\begin{tabular}{|c|c|}
\hline PROGRAMA & DESCRIPCIÓN \\
\hline \multirow{3}{*}{$\begin{array}{l}\text { Programa de } \\
\text { Bacteriología }\end{array}$} & $\begin{array}{l}\text { Programa de formación profesional en el área específica de la salud, } \\
\text { relacionada con seres humanos, animal, vegetal y medio ambiente. Su } \\
\text { función es diagnosticar, pronosticar, vigilar, controlar; promocionar la salud } \\
\text { y prevención de enfermedades. }\end{array}$ \\
\hline & Aplicación del proceso evaluativo: \\
\hline & $\begin{array}{l}\text { Concibe la evaluación como estrategias personalizantes y formativas que } \\
\text { evidencian la apropiación y construcción del conocimiento, en el desarrollo } \\
\text { de competencias y en la formación, mediante valoración por procesos a } \\
\text { través de la reflexión crítica permanente, en coherencia con los propósitos } \\
\text { de su formación. }\end{array}$ \\
\hline \multirow{3}{*}{$\begin{array}{l}\text { Programa de } \\
\text { Enfermería }\end{array}$} & $\begin{array}{l}\text { Tiene como objeto de estudio el cuidado individual y colectivo. Su eje } \\
\text { transversal es el conocimiento biológico, humano, ético-legal y científico. } \\
\text { Aborda fenómenos culturales, políticos y sociales de la persona. }\end{array}$ \\
\hline & Aplicación de proceso evaluativo: \\
\hline & $\begin{array}{l}\text { Se promueven estrategias evaluativas personalizantes y formativas donde } \\
\text { el propósito central es la formación integral de la persona. No se evalúan } \\
\text { materiales u objetos sino talentos humanos. }\end{array}$ \\
\hline
\end{tabular}

Fuente: Elaboración propia a partir de Trabajo de Grado en Contexto de la Educación Superior Universidad Católica de Manizales.

El Proyecto Educativo Universitario - PEU de la Universidad Católica de Manizales, establece los referentes teóricos que orientan las dinámicas académicas y administrativas, dando coherencia a cada una de las acciones y proyectos emprendidos por la comunidad, convirtiéndose en el principal instrumento de gestión para alcanzar las metas propuestas; es la carta de navegación que guía las acciones y proporciona los valores, criterios y directrices que orientan el cumplimiento de las funciones derivadas de la Misión Institucional (UCM, 2007).

En lo que respecta a México, una de las problemáticas que enfrentan sus instituciones de Educación Superior, son los bajos índices de Eficiencia Terminal. Según datos publicados en 2005 por el Consejo Nacional de Ciencia y Tecnología, la Asociación Nacional 
de Universidades e Instituciones de Educación Superior y por el Observatorio Laboral, el total de la matrícula a nivel superior fue de 1'865.475; del cual se observa que las carreras de las áreas de Ciencias Naturales y Exactas y Ciencias Agropecuarias no tienen un peso específico significativo, apenas concentra el $1,9 \%$ y $2,3 \%$ respectivamente, mientras que las Ciencias Sociales y Administrativas y las Ingenierías agrupan el $48,3 \%$ y $33 \%$, del total de la matrícula (López y Villa, 2006)

Otro estudio latinoamericano, fue realizado por Pulido (2007), en la Universidad Pedagógica Experimental Libertador de Caracas - Venezuela, intitulado Paradoja del proceso de evaluación de los alumnos de posgrado de la Universidad Pedagógica Experimental Libertador, caso: Instituto de mejoramiento profesional del magisterio, tuvo como propósito detectar la paradoja entre la teoría de la evaluación y la praxis del proceso de evaluación de los aprendizajes de los estudiantes de la maestria en gerencia educacional. La investigación se valió de diferentes métodos de recolección de datos, entre estos, la entrevista, envío de correo electrónico y revisión de fuentes primarias: registros de calificaciones, planes de evaluación e informes finales; los resultados permitieron evidenciar el desconocimiento sobre la teoría de la evaluación, su alcance y el momento oportuno de llevarla a cabo; se destaca negativamente esta situación, dado que se trata de estudiantes de posgrado.

En función de los resultados alcanzados en diferentes estudios, se estima que la actual generación de alumnos tendrá que cambiar de empleo de cinco a siete veces durante su vida laboral. El pensamiento crítico y el convertirse en aprendices a lo largo de la vida debería ser el factor decisivo en la educación. Insistir en que todo es medible promueve solo un, y quizás falso, sentido de responsabilidad (Buckman, 2007: 35-36).Igualmente, muchos profesores universitarios están alarmados por la falta de preparación de los alumnos que ingresan a la universidad, y lamentan el hecho de que en los cursos introductorios a menudo tienen que enseñar las habilidades -escritura, pensamiento crítico, matemáticas básicas- que esperarian que los alumnos hubieran adquirido antes de llegar a la universidad. Los alumnos son inteligentes y capaces, pero a menudo carecen de las habilidades que les ayuden a competir con éxito en el nivel universitario (Moreno, 2016).

Vale resaltar lo señalado por González (2017) sobre la educación universitaria en Latinoamérica destacando que en la actualidad las nuevas tecnologías de comunicación e información han impactado la educación flexibilizándola y masificándola, refiere una sociedad del conocimiento preocupada por la educación como una vía para el crecimiento personal y para mejorar la calidad de vida. Asimismo, en su reflexión el autor señala siete aspectos relevantes que se orientan a definir el papel que deben cumplir todos los actores del proceso educativo.

En este sentido, resalta el papel del estado como promotor de una educación integral, responsable de diseñar políticas coherentes y de asignar los recursos económicos necesarios, como segundo aspecto, la importancia de contar con una gestión educativa competente, el tercer punto de reflexión se enfoca en la calidad académica basada en las competencias y preferencias de los estudiantes, orientadas por estudios técnicos y vocacionales. 
Otro de los temas que toma para la reflexión,es la importancia de la participación de un equipo de trabajo para construir un currículo acorde con los requerimientos de la sociedad. Pertinentemente, el quinto aspecto, atiende las características del docente, el cual debe ser un profesional comprometido con el desarrollo social y consciente de su responsabilidad en el desarrollo de la sociedad. Adicional, el autor, sugiere la participación de los estudiantes como:

...responsables y conocedores de
que es lo que van a realizar en la
Universidad. Conscientes de que la
decisión de estudiar determinada
carrera fue fruto de la evaluación
de dos aspectos fundamentales
(Competencias naturales y gusto
por esa profesión seleccionada),
estudiantes conscientes que en el
desarrollo de su profesión va a tener
más importancia el conocimiento y
no la nota final que alcanzó en la
asignatur. (González, 2017, s/n)

Lo anterior, tiene relación con la evaluación formativa, los resultados deben ser visibles en un profesional preparado para la acción, capacitado para el hacer profesional, asunto que se refuerza en el último punto de reflexión, la experiencia laboral, la practica o la vivencia profesional se constituye en el cierre y en la demostración de las competencias adquiridas durante la formación de los profesionales universitarios.

\section{Normativas, procesos de educación y prácticas evaluativas en la universidad ecuatoriana}

En esta sección se precian normas, procedimientos y las prácticas evaluativas en el contexto ecuatoriano, precisando en lo que respecta a las páracticas evaluativas, casos concretos de realidades universitarias de dicho país.

\section{a. Normativa}

Puntualmente, la Constitución de la República del Ecuador (2008) enmarca en sus artículos 26 y 29 lo referente a la educación:

Art. 26.- La educación es un derecho de las personas a lo largo de su vida y un deber ineludible a inexcusable del Estado. Constituye un área prioritaria de la política pública y de la inversión estatal, garantía de igualdad e inclusión social y condición indispenable para el buen vivir. Las personas, las familias y la sociedad tienen el derecho y la responsabilidad de participar en el proceso educativo.

Art. 29.- El Estado garantizará la libertad de enseñanza, la libertad de cátedra en la educación superior, y el derecho de las personas de aprender en su propia lengua y ámbito cultural.

Concatenadamente, la Ley Orgánica de Educación Superior LOES (2018) expresa en sus artículos 93, 96 y 103 lo pertinente aPrincipio de calidad, aseguramiento interno de la calidad y a la evaluación de resultados de aprendizaje, al respecto:

Art. 93.- El principio de calidad establece la búsqueda continua, auto-reflexiva del mejoramiento, aseguramiento y construcción colectiva de la cultura de la calidad educativa superior con la participación de todos los estamentos de las instituciones de educación superior y el Sistema de Educación Superior, basada en el equilibrio de la docencia, la investigación e innovación y la vinculación con la sociedad, orientadas por la pertinencia, la inclusión, la democratización del acceso y la euidad, la diversidad, la autonomía responsable, la integralidad, la democracia, la producción de 
concoimiento, el diálogo de saberes, y valores ciudadanos.

Art. 96.- El aseguramiento interno de la calidad es un conjunto de acciones que llevan a cabo las instituciones de educación superior con la finalidad de desarrollar y aplicar políticas efectivas para promover el desarrollo constante de la calidad de las carreras, programas académicos; en coordinación con otros actores del Sistema de Educación Superior.

Art. 103.- Evaluación de resultados de aprendizaje de carreras y programas.Para efectos de evaluación de resultados de aprendizaje de carreras y programas se establecerá un examen $u$ otros mecanismos de evaluación para estudiantes del mismo periodo académico. Los procesos de evaluación se realizarán sobre los conocimientos y de ser necesario según el perfil profesional se aplicará sobre otras competencias. (...)

En tanto, en el Reglamento de Régimen Académico (2018), en su Art. 15 , se resume que la organización del aprendizaje se planificará incluyendo los siguientes componentes:

1. Componente de docencia: aprendizaje en contacto con el docente, conjunto de actividades individuales 0 grupales desarrolladas con intervención directa del docente, de forma presencial o virtual, sincrónica o asincrónica que comprende las clases, conferencias, seminarios, talleres, proyectos en el aula.

2. Componente de prácticas de aplicación y experimentación de los aprendizajes. Considerada como el conjunto de actividades individuales o grupales de apliacaión de contenidos procedimentales, conceptuales, entre otros, a la resolución de problemas prácticos, comprobación, experimentación, contrastación, replicación y demás que defina la IES.

3. Componente de aprendizaje autónomo. Conjunto de actividades indiviudales o grupales desarrolladas de forma independientepor el estudiante sin contacto con el personal académico o el personal de apoyo académico. Para su desarrollo deberán planificarse y evaluarse actividades específicas, tales como: lectura crítica de textos, investigación documental, escritura académica o científica, elaboración de informes, portafolios, proyectos, entre otras.

\section{b. Procesos de educación universitaria del Ecuador}

La formación universitaria en Ecuador tiene alrededor de 300 años de vigenciay ha otorgado a la sociedad profesionales en diversos ámbitos, elproceso de aprendizajeha sido canalizado de acuerdo a las realidades de cada época y, de manera cónsona, las prácticas evaluativasson equivalentes a los modelos y tendencias globales que acogen las universidades. En este sentido, el presente trabajo analiza las prácticas de evaluación aplicadas en la carrera de Administración de Empresas de la Universidad Estatal Península de Santa Elena (UPSE).

La UPSE se crea en el año 1998,enun sector turístico con una economía no muy favorable; con ausencia de referente de estudios universitarios. Actualmente la institución cuenta con 21 años de funcionamiento, se ofertan carreras como: Agropecuaria, Biología, Enfermería, Comunicación, Gestión y Desarrollo Social, Derecho, Electrónica y Automatización, Telecomunicaciones, Tecnologías de la 
Información, Contabilidad y Auditoría, Administración de Empresas, Gestión y Desarrollo Turístico, Educación Básica, Educación Inicial, Pedagogía de los Idiomas Nacionales y Extranjeros, Petróleos, Ingeniería Civil e Ingeniería Industrial, dicha oferta académica se ha ido ajustando a las necesidades de la provincia con la finalidad de impactar positivamente en los sectores productivos peninsulares.

Se toma de referencia 3 universidades del país para conocer como se desarrolla el proceso de evaluación como parte esencial del proceso de aprendizaje, esencialmente en las carreras de Administración de Empresas (Cuadro 2)

\section{Cuadro 2 \\ Universidades y procesos de aprendizaje}

\begin{tabular}{|c|c|}
\hline UNIVERSIDAD & CONDICIÓN \\
\hline $\begin{array}{l}\text { Universidad Técnica } \\
\text { Particular de Loja - } \\
\text { UTPL }\end{array}$ & $\begin{array}{l}\text { Procesos, recursos y metodologías de enseñanzas se desarrollan } \\
\text { bajo estándares de calidad que garantizan no solo la formación } \\
\text { integral y vanguardista de los estudiantes, sino su potencial } \\
\text { de inserción en un mundo global. A través de la cátedra de } \\
\text { Emprendimiento e Innovación UTPL, los estudiantes se forman para } \\
\text { emprender con éxito, impulsando ideas innovadoras y funcionales. }\end{array}$ \\
\hline $\begin{array}{l}\text { Universidad Técnica } \\
\text { Equinoccial de } \\
\text { Guayaquil - UTEG }\end{array}$ & $\begin{array}{l}\text { La metodología implentada por la UTEG comprende métodos } \\
\text { como presentación de problemas, análisis de casos, simulaciones, } \\
\text { actividades de aprendizaje cooperativo, etc. Prácticas autónomas } \\
\text { como investigaciones, estudios de proyectos, informes, etc. } \\
\text { Mantienen un sistema muy funcional de tutorías y apoyo al } \\
\text { estudiante. Conjunto de actividades sincrónicas y asincrónicas: } \\
\text { guías de estudios digitales, documentación digital, material } \\
\text { multimedia, webs de consulta, foros académicos, trabajos grupales, } \\
\text { videoconferencias, etc. }\end{array}$ \\
\hline
\end{tabular}

Se fomenta la implementación de oportunidades emprendedoras y evaluar sus posibilidades de éxito, en el diseño y evaluación de proyectos y en el análisis de los procesos relacionados con el

Universidad de Las funcionamiento de una organización, estableciendo estrategias de Américas - UDLA mejora y aseguramiento de la calidad. Se practica la metodología de casos/visitas de campo, simuladores de negocios. Docentes con experiencia empresarial. Universidad acreditada en Estados Unidos por WSCUC.

Fuente: https://inscripciones.utpl.edu.ec/file/1174/download?token=QIsc9SZg;https://www.uteg. edu.ec/licenciatura-en-administracion-de-empresas-online/;https://www.udla.edu.ec/carreras/ programas-academicos/pregrados/facultad-de-ciencias-economicas-y-administrativas/ingenieria-comercial_mencion-administracion-de-empresas-2/ 
c. Prácticas evaluativas aplicadas en la carrera de Administración de Empresas, la Universidad Estatal Península de Santa Elena (UPSE)

El proceso de investigación para analizar las prácticas evaluativas en la carrera de administración de empresas de la UPSE,consideró como población estudiantil en la carrera de
Administración de Empresas para el momento del estudio 621estudiabtes, de los cuales se tomaron en consideración a 175 pertenecientes a los semestres tercero y cuarto; es decir, un muestreo no probabilístico por conveniencia. Respecto a la población docente, se asume el censo, abordando el $100 \%$, respresentado por 28 docentes (tabla 1 )

\section{Tabla 1}

Población

\begin{tabular}{cllc}
\hline $\mathbf{N}^{\circ}$ & & Detalle & Cantidad \\
\hline 1 & Estudiantes & & 621 \\
\hline 2 & Docentes & & 28 \\
\hline & & TOTAL & 649 \\
\hline
\end{tabular}

Fuente: Elaboración propia a partit de la base de datos Carrera A.E.

Como procedimiento, se aplicaron encuestasenfísicoenaulas determinadas y salas de docentes específicas. Se consideraron momentos oportunos para su aplicación, particularmente en estudiantes, previo a rendir exámenes de cierre de ciclo, evitando la influencia de factores anímicos o indisposiciones. El cuestionario para estudiantes y docentes estuvo compuesto por 20 items. Las categorías planteadaspor preguntas se definieron como: Nunca, En Ocasiones y Siempre. Se logró obtener información desde la planificación, ejecución y el propio control del proceso evaluativo.

Tomando como referencia lo anterior, el currículo de la carrera de Administración de Empresas, se ha ido adaptando acorde a los resultados de los estudios prospectivos de la demanda ocupacional, cohorte a cohorte se han mejorado, implementado y ajustado las prácticas evaluativas con la finalidad de perfeccionar y garantizar los procesos de aprendizaje del futuro profesional. De igual manera, las prácticas evaluativas han evolucionado pasando por diversos niveles y alcanzando diferentes objetivos adoptado aquellos con los mejores resultados.

En los inicios de la carrera, en el año 2007 , las primeras prácticas o estrategias evaluativas que fueron identificadas en la planificación académica y aplicada por los docentes fueron: Evaluaciones escritas, actuaciones, exposiciones, ejercicios en clases, tareas, aprendizaje basado en problemas, trabajo de campo, entre otras.

Estas prácticas evaluativas se mejoraron con el tiempo, por los efectos de actualización y globalización educativa; no obstante, algunas se mantienen y otras se han incorporado como resultado de los nuevos sistemas formativos impulsados por el campo empresarial, las que se han incorporadon son: Portafolio, ensayo, 
rúbricas, organizadores gráficos, guías de observación, informes, prácticas de laboratorios, trabajo autónomo, proyectos, principalmente.

Los resultados de las nuevas o mejoradas prácticas evaluativas, han permitido que los estudiantes logren un mayor acercamiento con la realidad laboral. No sólo se conoce el elemento cognitivo de la ciencia sino que se llega a la práctica real y propia de su formación profesional; dejando de lado la simulación o supuestos, con los que se venía educando a través del tiempo.

Sobre la evalaución educativa, Balam (2009), señala que una de las dimensiones más relevantes está referida a todas aquellas técnicas, instrumentos y procedimientos que suelen utilizarse en las distintas prácticas educativas, las que se clasifican en, (diagrama 1):

\section{Diagrama 1 \\ Técnicas de Evaluación}

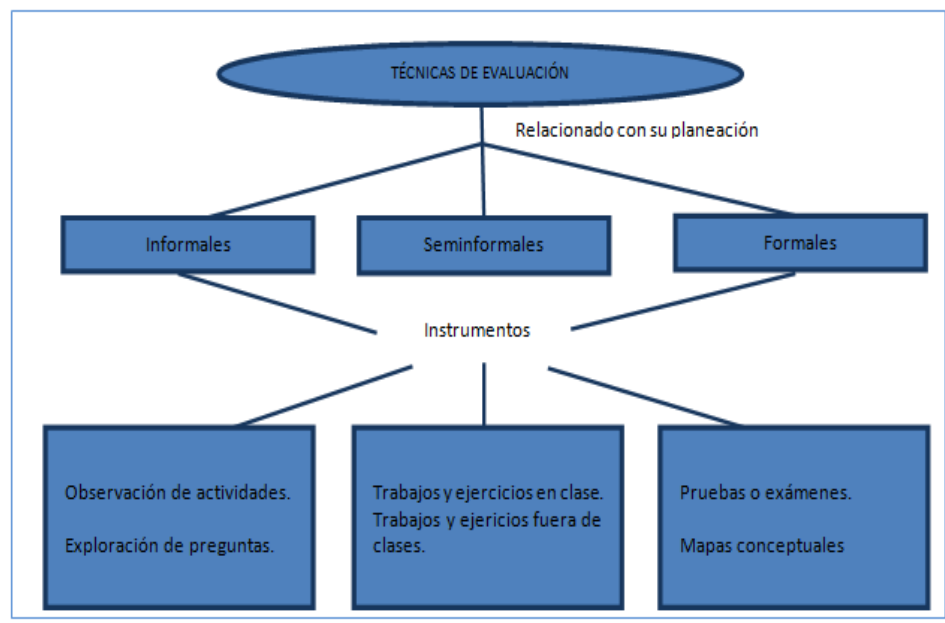

Fuente: Elaboración propia

Con respecto a las técnicas Informales, resaltan: a) Observación de actividades realizadas por alumnos, técnica utilizada por el profesor incidencialmente y cuando alumnos aprenden de manera autónoma. Se dirige atención a otros aspectos del estudiante como: habla, habilidades, estrategias, razonamiento. Utilización de instrumentos como registros anecdóticos, listas de control, diario de clase; y b) Indagación durante la clase.

El autor incluye las técnicas semiformales, representadas por: a) Trabajos y ejercicios en clases, más preparadas que las anteriores, demandan mayor tiempo de valoración y respuestas más duraderas, entre ellas están: Trabajos y ejercicios realizados en clases, alineados a objetivos de aprendizaje y permitiendo decisiones para regulación interactiva, retroactiva 
o proactiva; y b) Tareas y trabajos autónomos, suelen ser muy variados y de forma individual o cooperativo; además de planearse correctamente. La información obtenida debe socializarse oportunamente, explicando procesos correctivos.

Adicionalmente, con respecto a las técnicas formales, se precisa que estas demandan procesos de planificación más definidos y elaboración sofisticada, incluyendo en ellas: a) Pruebas o exámenes: que pueden ser de dos tipos; los estandarizados, elaborados por especialistas o también los formulados por los propios profesores, evaluaciones basadas en normas o criterios. Estos exámenes pueden más bien medir capacidades generales y no habilidades o conocimientos específicos, dejando de lado fallas o posibilidades de retroalimentación; y, b) Mapas conceptuales, solicitado de acuerdo a propuestas del docente en función de tema general, a partir de conceptos propuestos, facilitando estructuras sobre temas determinados e insumos necesarios.

La administración como toda área profesional tiene sus particularidades, por ende no toda estrategia evaluativa puede ser aplicada, se requiere del cumplimiento de parámetros e indicadores relacionados con la consecución de resultados de aprendizaje de acuerdo a las unidades de organización del aprendizaje. Para citar ejemplos en la carrera de Administración de Empresas de la UPSE, las estrategias relevantes según informes de fin de ciclo en cada periodo académico, destacan (cuadros $3,4,5$ y 6 ):

- Proyectos - Examen final

\section{Cuadro 3 Consideraciones de Proyectos}

\begin{tabular}{ll}
\hline \multirow{2}{*}{$\begin{array}{ll}\text { Procedimientos } \\
\text { Generales }\end{array}$} & Fundamentación legal \\
\cline { 2 - 2 } & Definición de cátedras integradoras y proyectos por semestres \\
\cline { 2 - 2 } & Protocolos o diseños de investigación \\
\cline { 2 - 2 } & Consideraciones generales \\
\hline
\end{tabular}

Fuente: elaboración propia 


\section{Cuadro 4 \\ Procedimientos}

\begin{tabular}{|c|c|}
\hline \multirow{11}{*}{$\begin{array}{l}\text { Procedimientos } \\
\text { de Asignaturas }\end{array}$} & Elaboración de programas de estudio \\
\hline & Diseño del proyecto según necesidades del semestre \\
\hline & Socialización con estudiantes \\
\hline & $\begin{array}{l}\text { Definición de ideas o propuestas por parte de estudiantes } \\
\text { (búsqueda de necesidades }\end{array}$ \\
\hline & Desarrollo del diseño durante el semestre \\
\hline & Vistas empresariales \\
\hline & Salidas de campo \\
\hline & Revisión de borradores \\
\hline & Elaboración de prototipos \\
\hline & Verificación de itegración de asignaturas del semestre \\
\hline & Exposición y defensa de proyectos \\
\hline
\end{tabular}

Fuente: elaboración propia

\section{Cuadro 5}

Parámetros de Rúbrica

\begin{tabular}{|c|c|c|c|}
\hline \multirow{16}{*}{ Rúbrica } & Parámetro & Indicador & Puntaje \\
\hline & \multirow{3}{*}{$\begin{array}{l}\text { Aspectos } \\
\text { generales }\end{array}$} & Imagen & 2 \\
\hline & & Vestimenta & 2 \\
\hline & & & 4 \\
\hline & \multirow{5}{*}{$\begin{array}{l}\text { Documento } \\
\text { impreso }\end{array}$} & Entrega puntual & 2 \\
\hline & & Esquema presentación & 2 \\
\hline & & Redacción & 2 \\
\hline & & Ortografía & 2 \\
\hline & & & 8 \\
\hline & \multirow{6}{*}{ Defensa } & Trabajo en equipo & 4 \\
\hline & & Dominio del tema & 4 \\
\hline & & Dominio escénico & 4 \\
\hline & & Claridad y precisión en la exposición & 6 \\
\hline & & Claridad y precisión en respuestas & 10 \\
\hline & & & 28 \\
\hline & & Puntaje final & 40 \\
\hline
\end{tabular}

Fuente: elaboración propia 
- Ejemplo de asignatura

\section{Cuadro 6 Ejemplificación de la evaluación procesual}

\begin{tabular}{ll}
\hline & Exposiciones y memorias \\
\cline { 2 - 2 } $\begin{array}{l}\text { Evaluación } \\
\text { procesual }\end{array}$ & Palleres participativos/colaborativos \\
\cline { 2 - 2 } & Prueba de ejecución de tarea: converstorio o coloquio/panel integrado \\
\cline { 2 - 2 } & Análisis crítico de texto selecto \\
\cline { 2 - 2 } & Ensayo \\
\hline
\end{tabular}

Fuente: elaboración propia

Otra cuestión importante de la evaluación. Hay finalidades pedagógicamente ricas (comprender, mejorar, aprender, motivar, diagnosticar...), pedagógicamente pobres (medir, comparar, clasificar, seleccionar...) y finalidades espurias (jerarquizar, controlar, imponer, castigar...). Habría que potenciar las funciones pedagógicamente ricas frente a las pobres, y habría que erradicar las funciones espurias (Moreno, 2016)

\section{Proceso de aprenidazaje en carreras de administración de empresas del Ecuador}

Se muestra en esta sección la percepción del docente y del estudiante de la carrera de administración del Ecuador, como sigue:

- Percepción Docente frente a la evaluación del aprendizaje: Análisis Descriptivo

Para los docentes de la carrera de Administración de Empresas, las prácticas evaluativas que se llevan a cabo en el proceso de aprendizaje tienen algunos rasgos particulares en función de los elementos indagados (Gráfico 1). Desataca pregunta 16 que alcanza el primer lugar con $93,31 \%$, referida a la Transparencia en las calificaciones, según los docentes los estudiantes tienen pleno conocimiento de los criterios de valoración en las estrategias aplicadas.

En segundo lugar, los docentes (90 $\%$ ) expresan que dan a conocer a sus estudiantes los Objetivos a evaluar en el proceso de enseñanza aprendizaje. Por su parte, con similar porcentaje $(88,70 \%)$, las respuestas a las preguntas 11,20 y 8 , resultando que en la percepción de los académicos el Proceso de evaluación y sus Funciones, buscan una potenciación en el aprendizaje. En cuanto a respuestas de la pregunta 8 se revela que la Actitud, métodos y procedimientos, estimulan la confianza y el estudio en los estudiantes. Con poca diferencia $(87,87 \%)$, las respuestas a las preguntas 2 y 10, dejan ver que los docentes Socializan con los estudiantes los criterios de evaluación y además que, la evaluación se centra en la comprobación y verificación de lo aprendido.

De manera general, los docentes 


\section{Gráfico 1}

\section{Encuesta a docentes}

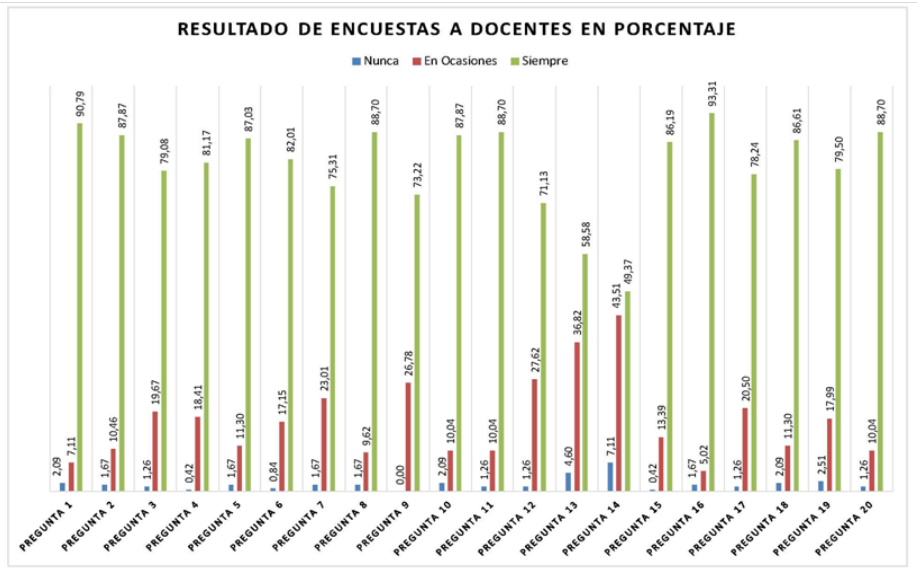

Fuente: elaboración propia

coinciden que Siempre dan las debidas orientaciones, conocimientos e instrucciones para el proceso de evaluación y que se busca, a más de la participación, el empoderamiento de los conocimientos y experiencias transmitidas. A continuación se presenta el gráfico 2 del método multivariado, análisis de clasificación (cluster), cuya distribución de datos muestra una asimetría Izquierda:

\section{Gráfico 2}

Cluster, Distribución de datos - Docentes

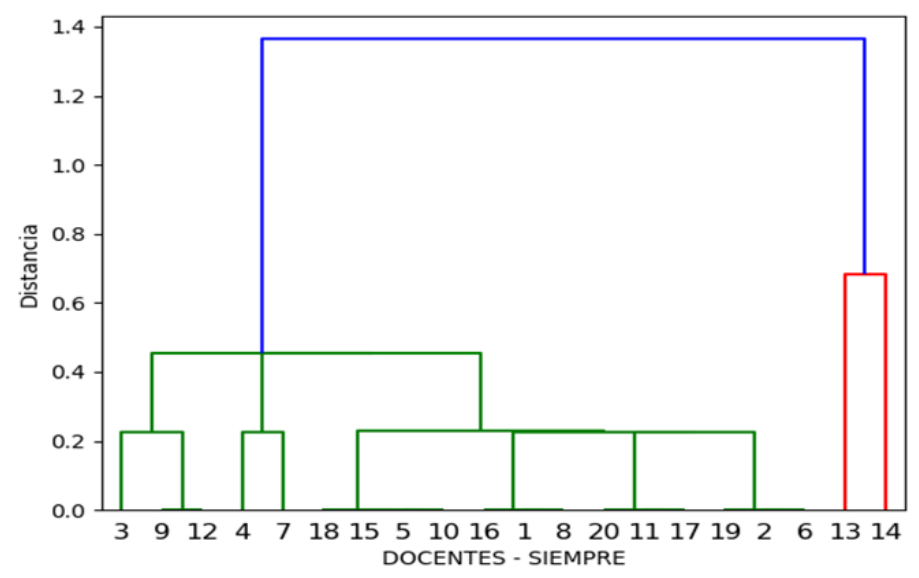

Fuente: elaboración propia 


\section{Percepción de los estudiantes frente a la evaluación del aprendizaje. Análisis descriptivo Gráfico 3 Encuesta a estudiantes}

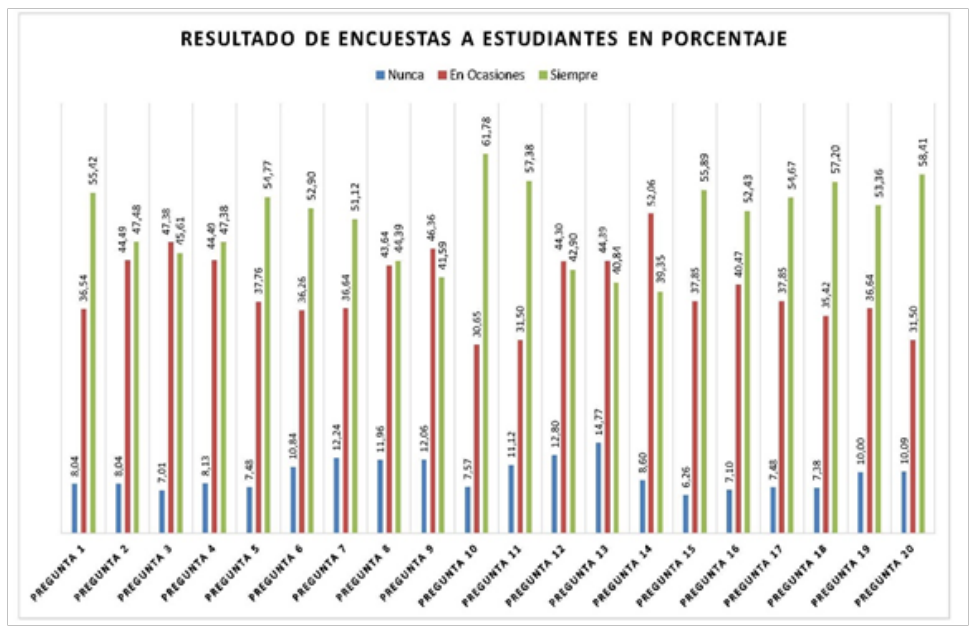

Fuente: elaboración propia

Desde el punto de vista de los estudiantes de la carrera de Administración de Empresas, los resultados de las prácticas evaluativas que se llevan a cabo en el proceso de aprendizaje tienen resultados que tienden a ubicarse en un porcentaje medio. Entre las respuestas destaca la percepción del $61,78 \%$ de que la evaluación a la cual son sometidos como estudiantes se centra en la comprobación, verificación y medición de lo aprendido. Convenientemente, $58,4 \%$ considera que la Evaluación tiene un efecto educativo,mientras, $57,38 \%$, reconoce que la evaluación permite la Potenciación del aprendizaje en concordancia con la opinión de los docentes. Para un poco más de la mitad de la muestra, $57,20 \%$, los docentes cumplen con la socialización de los resultados de las evaluaciones. Por otro lado, 55,89 \% reconoce que la calificación de las evaluaciones se ejecuta en base a criterios e indicadores.

La novedad presentada con los dos sujetos participantes de esta investigación se debe que los resultados de los estudiantes reflejan un valor máximo del $61,78 \%$, disminuyendo apenas entre 1 o 2 puntos porcentuales en la ubicación ordinal. Se observa además que, dentro de las categorías planteadas; las respuestas se enmarcan no solo en la condición de Siempre, existen marcadas tendencias con la alternativa de En Ocasiones. Identificando que no siempre los estudiantes, están satisfechos con los rasgos del proceso evaluativo.

A continuación se presenta el gráfico4 del método multivariado, análisis de clasificación (cluster), cuya distribución de datos muestra una tendencia Acampanada: 


\section{Gráfico 4}

Cluster, Distribución de datos - Estudiantes.

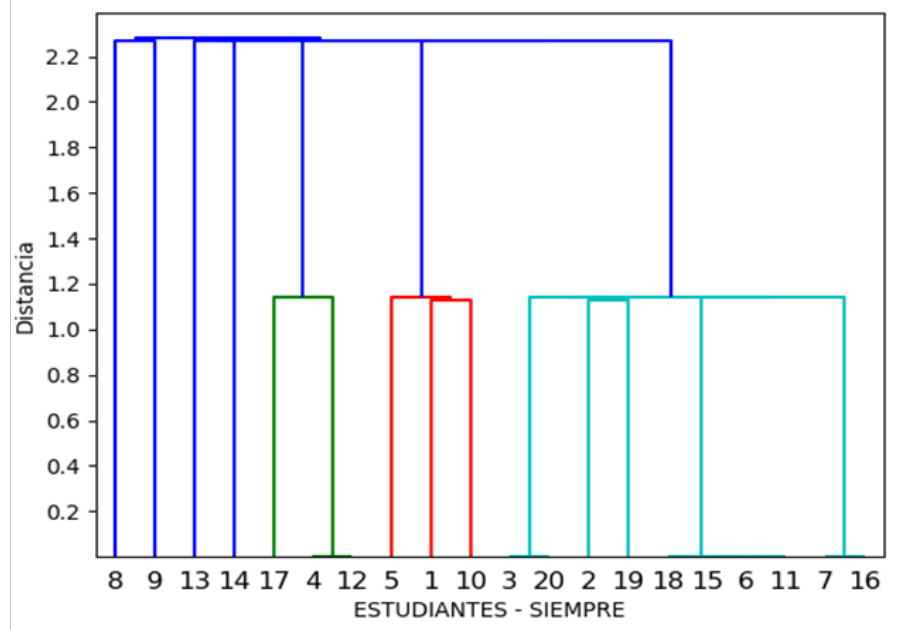

Fuente: elaboración propia

- Análisis Correlacional entre Docentes y Estudiantes, a partir de las categorías negativas del instrumento.

\section{Gráfico 5}

Tendencias negativas, docentes - estudiantes.

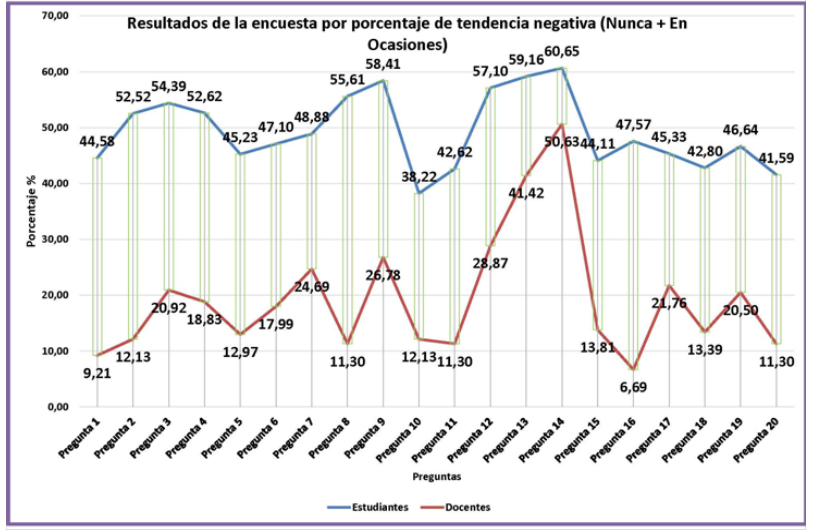

Fuente:elaboración propia 
Los resultados obtenidos de la aplicación del instrumento, respecto a las categorías: Nunca y En Ocasiones, tienen su peculiaridad de acuerdo a:

a) Existe una marcada dispersión entre percepción de docentes versus percepción de estudiantes. La sumatoria entre los Nunca y En Ocasiones para docentes, tiene una tendencia que va desde $9 \%$ hasta un $29 \%$. Con excepción de las preguntas 13 y 14 que llegan al $41,42 \%$ y $50,63 \%$, respectivamente. Las respuestas son una negación a la aplicación de Evaluaciones con temas distintos o de otras disciplinas; O, mayoritariamente No se aplican heteroevaluaciones, coevaluaciones ni autoevaluaciones.

b) Para los Estudiantes la tendencia es alta en las categorías de Nunca y En Ocasiones. Sus rangos porcentuales van desde $38 \%$ hasta $61 \%$; sobrepasando la categoría de Siempre. Estos resultados no se corresponden con la percepción de los Docentes; evidenciando por tanto, no estar de acuerdo con varios procedimientos.

c) De los resultados obtenidos de los estudiantes se destaca que Las evaluaciones cumplen su función de comprobación, verificación y medición. Para la pregunta 14, los sujetos intervinientes coinciden que por lo general No se aplican heteroevaluaciones, coevaluaciones ni autoevaluaciones.

\section{Conclusiones}

Las prácticas evaluativas se constituyen en un instrumento importante dentro del proceso de enseñanza aprendizaje.Tantoel recorrido por las teorías como lo señalado en algunas de las experiencias latinoamericanas descritas, confirmaron lo esfuerzos y avances que sobre el tema se llevan a cabo en las instituciones de educación superior. Asimismo, se evidenciaron las tendencias y debilidades que presenta la evaluación, en cuanto a sus objetivos y diseño.

En cuanto a la experiencia de la carrera de Administración de Empresas en la UPSE, el currículo académico se ha adaptado a los cambios progresivos del entorno peninsular, sus malla curricular se ajusta y actualiza en función de los estudios prospectivos que se aplican en conjunto con el sector empresarial, además de dar cumplimiento a las reformas legales que el país dispone.

De igual forma, al interior de la academia, la carrera de Administración de Empresas, busca los medios y herramientas más apropiadas, a partir de los resultados que reflejan las evaluaciones del proceso académico en cada periodo, paraimpulsar desde cada asignatura la transmisión de conocimiento y la mejora de las prácticas evaluativas, principalmente buscando el ejercicio reflexivo y critico que puede otorgar las experiencias y vivencias durante la formación.

Específicamente, los resultados del estudio para analizar la percepción de docentes y estudiantes sobre las practicas evaluativas en el proceso de aprendizaje dela carrera de administración deempresas, dio como principal resultado que la evaluación, independientemente de su mecanismo y los hechos que se presenten, logra un efecto educativo de mejora en los estudiantes, a pesar de que existen diferencias entre la percepción de los estudiantes y la de los docentes.

Por los resultados mostrados, se hace necesario una revisión profunda de los instrumentos y los objetivos de la evaluación que se aplican en la diferentes 
asignaturas de la carrera, tomando en consideración que en la medida que los instrumentos de investigación sean mejor diseñados y orientados a la evaluación formativa, se logrará una mayor motivación y compromiso por parte de los estudiantes $\mathrm{y}$, consecuentemente, mejor rendimiento y resultados, alejados del cumplimiento de un requisito $y$ mas cercano a la excelencia. Para los docentes, una revisión de las practicas evaluativas implica el reto de abordar insumos nuevos, salir de lo cotidiano y estar a la paridad de lo que vive el mundo empresarial.

Finalmente, la evaluación es un instrumento y en su aplicación se deben conjugar instancias donde se puedan identificar bases teóricas pero a la vez, es imperioso que contemplen el reconocimiento de una realidad; es decir, pasar al proceso de experimentación que puede darse, por ejemplo, a través del trabajo autónomo. Tal como se mencionó debe tener un enfoque progresivo $y$ un encadenamiento de instrumentos que sirvan de ayuda tanto a docentes como estudiantes, promoviendo una evaluación formativa y educación para la vida.

\section{Referencias bibliográficas}

Álvarez Méndez, Juan (2006), La evaluación educativa al servicio de quien aprende: el compromiso necesario con la acción crítica. Miño y Dávila.Madrid, España

Ahumada, Pedro (2001), La evaluación en una concepción de aprendizaje significativo. Valparaiso: Universitarias, 2001.

Balam Güemez (2009), Una caracterización de las prácticas evaluativas en cursos de Álgebra de Nivel Su- perior. (Tesis de pregrado inédita), Universidad Autónoma de Yucatán, Yucatán, México.

Barberá, Elena (2003), Estado y tendencias de la evaluación en educación superior. Red Estatal de Docencia Universitaria, $N^{\circ} 2($ Vol.3), 94-99

Barrón, Marìa (2009), “Docencia universitaria y competencias didácticas", Perfiles Educativos. Vol. XXXI, núm. 125: 76-87

Boud, David. (2007). Reframing assessment as if learning were important. Rethinking Assessment in Higher Education: Learning of the longer term. Londres y Nueva York: Routledge, 14-25

Boud, David y Falchikov, Nancy (2010), Retbinking Assessment in Higber Education. Learning for the longer term. London: Routledge.

Buckman, Ken (2007), "What Counts as Assessment in the 21st. Century? Thought\&Action", en The New HigherEducation Journal, Otoño, 29-37

Asamblea Nacional Constituyente (2008), Constitución de la República del Ecuador. 2008, Ecuador, Asamblea Nacional, 20 de octubre 2008.

Duque, Paula; Henao, Olga; Palacios, Nubia (2017), Prácticas evaluativas en el contexto de la Educación Superior. (Tesis de pregrado inédita), Universidad Católica de Manizales, Manizales, Colombia.

González, Orlando (2017), Reflexión sobre la educación universitaria en América Latina, Centro de Estudios Latinoamericanos.Recuperado de https://celu.co/reflexion-la-educacion-superior-america-latina/

Hargreaves, Andy y Dennis Shirley (2012), La cuarta vía. El promete- 
dor future del cambio educativo. Barcelona: Octaedro

Hernández Mario (2016), Prácticas evaluativas del aprendizaje en la Universidad Deportiva del Sur de Venezuela. Revista Cubana de Educación Superior, 2016(2), 4-15

Ibarra, Maria y Rodríguez, Gregorio (2010), Aproximación al discurso dominante sobre la evaluación del aprendizaje en la Universidad. Revista de Educación, 351(16), 385407. Recuperado de http://www.revistaeducacion.educacion.es/re351/ re351_16.pdf

Asamblea nacional de la republica de Ecuador (2018), Ley Orgánica de Educación Superior del Ecuador. 2018, Ecuador, Asamblea Nacional, Año II - No 297, jueves 2 de agosto.

Laboratorio Latinoamericano de Evaluación de la Calidad Educativa. LLECE. Evaluación y Prácticas Pedagógicas en Ciencias Naturales. Resultados de las Pruebas de CC, UNESCO, Santiago (2008) NN en $6^{\circ}$ de Primaria.

López, Rogelio. y Villa, Juan (2006), Estructura de la formación de profesionales $e$ investigadores por áreas del conocimiento, Jornadas anuales de investigación. Recuperado de http://www.ceiich.unam.mx/ pdfeacad/jornada/torres.html

Moreno Tiburcio (2016), Evaluación del aprendizaje y para el aprendizaje. Reinventar la evaluación en el aula. Cuajimalpa, México: Universidad Autónoma Metropolitana

Nieto, Santiago (2010), El discurso del profesorado universitario sobre la evaluación del aprendizaje de los alumnos como estrategia de innovación y cambio profesional: expo- sición y análisis de una experiencia. Revista de Educación, 322, 305324. Recuperado de http://www.revistaeducacion.educacion.es/re351/ re351_16.pdf

Organización de las Naciones Unidas para la Educación, la Ciencia y la Cultura. UNESCO. (2018), Learning Portal. Recuperado de https:// learningportal.iiep.unesco.org/es/ fichas-praticas/improve-learning/escuelas-y-aulas/evaluacion-formativa

Pulido, Jesús (2007), Paradoja del proceso de evaluación de los alumnos de postgrado de la Universidad Pedagógica Experimental Libertador, caso: instituto de mejoramiento profesional magisterio Investigación y Postgrado. Universidad Pedagógica Experimental Libertador Caracas, Venezuela, vol. 22, núm. 2. pp. 87114

El Consejo De Educacion Superior (2018), Reglamento de Régimen Académico del Ecuador. 2018, Ecuador, El Consejo De Educacion Superior, No. 051-2013 25-ene.-2017

Turpo, Osbaldo (2013), Posicionamiento de los docents de ciencias en la evaluación de los aprendizajes: una aproximación a sus objetividades. Evaluación Educativa, 24(2), 230-236. Recuperado de https:// reader.elsevier.com/reader/sd/pii/ S0187893X13724674?token=5CC043C4E6D1B273FB83A1C3 D 836C6D3D47A 796 B 91161829427CA956E629775F$37 \mathrm{~B} 33151 \mathrm{~F} 3168328 \mathrm{C} 1130 \mathrm{~A}-$ 107294D7F3

Universidad Católica de Manizales. (2007-2013). Marco Teleológico de la Universidad Católica de Manizales: Manizales. Centro Editorial UCM.

- Esta obra está bajo una licencia de Creative Commons Reconocimiento-NoComercial- Compartirlgual 3.0 Unported. http://creativecommons.org/licenses/by-nc-sa/3.0/deed.es_ES 